\title{
Viability of In-Service, Low-Cost and Spatially Unambiguous OTDR Monitoring in TDM- and WDM-PON Access Networks
}

\author{
L. Costa ${ }^{1}$, J. A. Lázaro ${ }^{2}$, V. Pólo ${ }^{2}$, A. Teixeira ${ }^{1}$ \\ ${ }^{1}$ Instituto de Telecomunicações, 3810-193 Aveiro, Portugal \\ ${ }^{2}$ Universitat Politécnica de Catalunya, 08034 Barcelona, Spain \\ Tel: (351) 234377 900, Fax: (351) 234377 901, e-mail: liliana.nicolau@ua.pt
}

\begin{abstract}
A new strategy is proposed to efficiently monitor fibre plant failures in current and future optical access networks by implementing an OTDR subsystem in the physical layer. The viability of the method is evaluated by analyzing its theoretical performance limits. Experimental results demonstrate the applicability of the method.

Keywords: monitoring, optical time domain reflectometry, next generation access networks.
\end{abstract}

\section{INTRODUCTION}

Optical time domain reflectometry (OTDR) has been reported in literature as the most popular choice to detect and locate failures in passive optical networks (PONs). In the context of next-generation access networks, where longer reach and higher number of users are supported, time and cost constraints on finding the cause of a problem are even more stringent. Therefore, one needs a way of automatically detect fibre plant failures in the physical layer of the network as quickly as possible and without disrupting traffic. Several in-service OTDR methods have been proposed to solve this problem. Embedded solutions are attractive [1] because they re-use the transmitter and/or receiver hardware and avoid the use of extra components in the optical path. However, like other in-band approaches, they are restricted to limited time operating windows defined by data transmission. Coherent OTDR gives the best possible receiver sensitivity and is consequently capable of monitoring longer fibre distances [2], but it has higher costs and complexity, since a highly coherent laser source is needed.

In this paper we propose an in-service and out-of-band approach, based on a cheap and tuneable OTDR laser source and a set of spectrally efficient reflective elements located in key intermediate and terminal locations of the network. These reflective elements create distinct events in the OTDR trace and give a distinct path signature in both wavelength and time domains for each fibre span or branch. We analyze the performance limitations of the proposed method according to the maximum measurable distance for different values of OTDR's launch power and the influence in the signal-to-noise ratio (SNR) at the receiver. We also study the maximum number of reference reflective elements according to typical access network topologies. An experimental proof-ofconcept shows that technological and performance constraints may be significantly relaxed if this new OTDR approach is used to monitor fibre failures in PONs.

\section{FUNDAMENTAL PERFORMANCE LIMITS OF PROPOSED OTDR MONITORING STRATEGY}

Well-known limitations of conventional OTDR with direct-detection, like the trade-off between spatial resolution and dynamic range can be overcome if we monitor the presence of reflective events created by passive reflectors, which can be mirrors, couplers or fibre Bragg gratings (FBGs), placed at key network points. This strategy has been used in [3] and [4]. Using a single-wavelength OTDR to monitor tree-structured PONs [3] may lead to ambiguity in failure detection, if some of its fibre branches have the same length. Relying on the measurement of attenuation variations of reflective events which are located at the same distance is not a good solution, since aging or upgrading operations can easily change the losses related with the other optical components present in an optical path or if the monitoring signal suffers amplification. A tuneable OTDR, together with reflective elements distinct in wavelength, is the way to surpass this problem. However, tuneable laser sources are expensive and allocating a wavelength for each reflective element implies a large monitoring wavelength band. The proposed OTDR strategy is based on spectrally efficient reflective elements, namely fibre Bragg gratings (FBGs) with distinct reflection spectrum characteristics, and enables the use of a low-cost laser source, a coarse DFB laser tuneable by temperature. An analysis of the theoretical limitations of the proposed OTDR monitoring strategy is performed. The fundamental limitations, related also to conventional OTDR, are mostly associated with the signal-to-noise ratio (SNR) of the backscattered and reflected OTDR signal. Following the mathematical simplification given in [5], we can establish the dependence of the SNR with the fibre length and also with measurement time. The expression used to calculate the initial SNR, without averaging, is given by:

$$
\begin{gathered}
S N R_{N A}=20 \log _{10}\left(\operatorname{Resp} P_{R}\right)-P_{D}-2 \alpha L \\
P_{R}=\frac{1}{2} \alpha_{S} S v_{g} W P_{i n}
\end{gathered}
$$




$$
P_{D}=20 \log _{10}(N E P \sqrt{B})
$$

where $P_{R}$ is the backscattered power, $P_{D}$ is the minimum detectable power by the receiver, $\alpha$ is the fibre attenuation coefficient, $\alpha_{S}$ is the Rayleigh scattering loss coefficient, $L$ is the fibre length, $S$ is the Rayleigh recapture coefficient and $v_{g}$ is the light group velocity. We consider an OTDR with the following characteristics: receiver based on a PIN photodiode with responsivity Resp $=0.95$, receiver bandwidth $B=100 \mathrm{MHz}$, noise equivalent power $N E P=0.2 \mathrm{pW} \cdot \mathrm{Hz}^{-1 / 2}$, and a light source with variable launch power $P_{i n}$, operating with a pulse width $W=1 \mu \mathrm{s}$ and based on a DFB laser tuneable by temperature within a short spectral band centred at $1625 \mathrm{~nm}$. In Fig. 1a it is shown the dependence of the initial SNR without averaging with the fibre length. Fig. $1 \mathrm{~b}$ shows the value of time needed to get an SNR equal to zero by using digital averaging. This condition is related with the OTDR's maximum reachable one-way distance in optical fibre and is marked in Fig. 1a as a dashed purple line. It means that to reach higher distance values one must use averaging or other signal processing technique to increase the SNR and be able to get proper OTDR traces. For example, with a launch power of $-10 \mathrm{dBm}$ the maximum measurable distance is about $12 \mathrm{~km}$ and it would take about $30 \mathrm{~s}$ to increase the spatial range to about $70 \mathrm{~km}$.

The time needed to perform averaging, and obtain an improved $S N R_{i m p}$, is calculated using the following mathematical expressions:

$$
\begin{gathered}
S N R_{i m p}=20 \log _{10}\left(\sqrt{N_{a v}}\right) \\
\text { Time }[s]=\frac{N_{a v}}{f_{\text {Prmax }}}=\frac{2 \times \text { Range }[m] \times N_{a v}}{\mathrm{v}_{\mathrm{g}}}
\end{gathered}
$$

where $N_{a v}$ is the number of additions to calculate the average and $f_{\operatorname{Pr} \max }$ is the maximum pulse repetition frequency which is defined according to the necessary OTDR's distance range.

Considering the addition of reflective elements, we can determine the maximum number of FBGs for two possible deployment scenarios: (i) FBGs are placed in series between fibre spans, which can constitute a single feeder fibre or a fibre ring; (ii) FBGs are placed in parallel, for example, at the end of the branches of a treestructured network; (iii) FBGs are placed both in series and in parallel to be adapted to a more complex network topology.

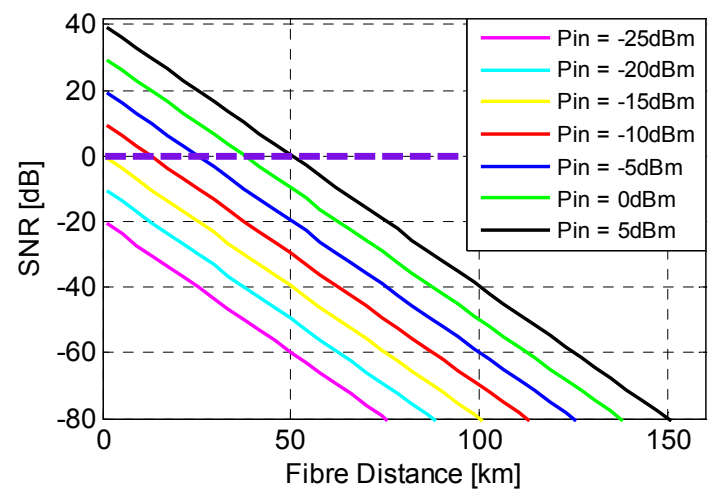

(a)

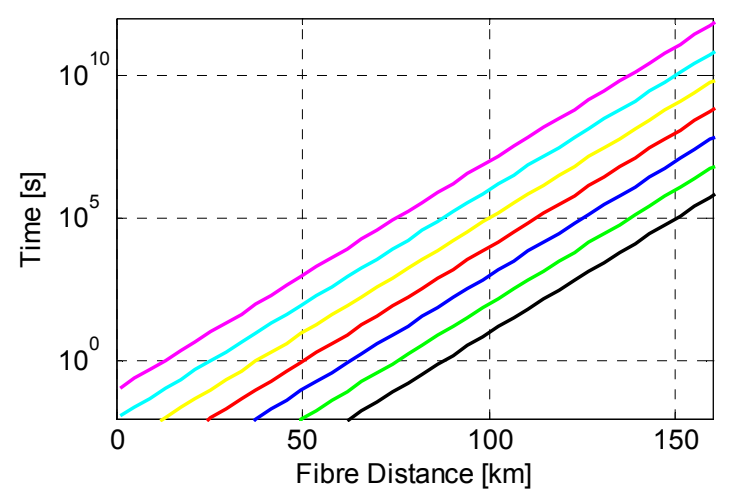

(b)

Fig. 1. (a) Initial SNR versus fibre distance and (b) averaging time versus fibre distance, for different values of OTDR's launch power $P_{\text {in }}$.

The first two types of deployment scenarios, which are more commonly used for real networks, are illustrated in Fig. 2a. If we consider again initial SNR equal to zero, the mathematical expressions which define the maximum allowable number of FBGs are respectively:

$$
\begin{gathered}
2 \alpha \sum_{i=1}^{N} L_{i}=20 \log _{10}\left(\operatorname{Resp} P_{R}\right)+10 \log _{10}(R)-P_{D}-2 \sum_{i=1}^{N-1} \beta_{R N} \\
2 \alpha\left(L_{F}+L_{B i}\right)=20 \log _{10}\left(\operatorname{Resp} P_{R}\right)+10 \log _{10}(R)-P_{D}+2 \times 10 \log _{10}\left(\frac{1}{N}\right)
\end{gathered}
$$

where $L_{i}$ is the length of the ith fibre span, $R$ is the FBG reflectivity (which is considered to be constant), $\beta_{R N}$ is the insertion losses resulting from by-passing the RNs, $N$ is the number of RNs which is equal to the number of FBGs, $L_{F}$ is the feeder fibre length and $L_{B i}$ is the length of the branch to monitor. In Fig. $2 \mathrm{~b}$ the maximum number of FBGs is shown for the case (i), corresponding to equation (6), with $\beta_{R N}=0.8 \mathrm{~dB}$ and $P_{i n}=0 \mathrm{dBm}$. 


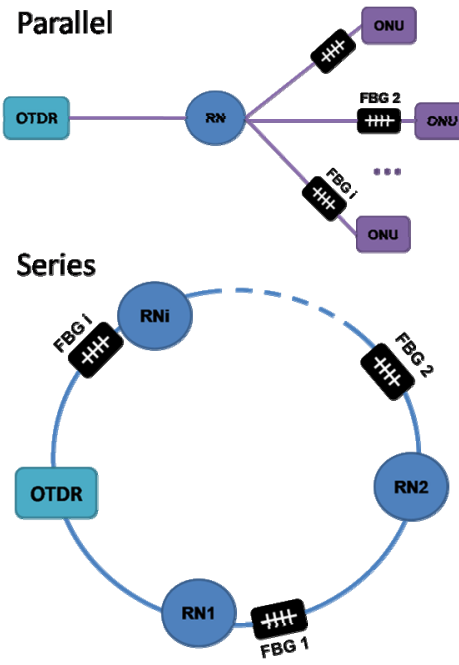

(a)

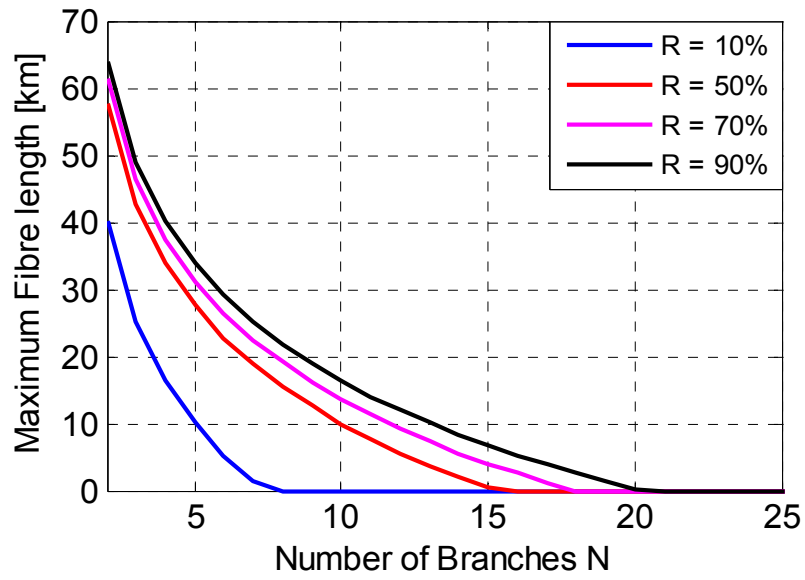

(b)

Fig. 2. (a) Deployment scenarios for proposed OTDR monitoring implementation: FBGs in parallel (top); FBGs in series (bottom). (b) Maximum fibre distance versus number of fibre spans, with FBGs placed in parallel. $R$ denotes the reflectivity of the gratings.

\section{EXPERIMENTAL VALIDATION}

The experimental setup is shown in Fig. 3. It is based on SARDANA network architecture, with a WDM ring plus TDM-PON trees, with remotely amplified RNs [6]. The experiments were carried out on the C-band, since this does not invalidate the proof-of-concept. The FBGs used are uniform and have the following characteristics: FBG1 has a central reflection wavelength equal to $1551.4 \mathrm{~nm}$, a FWHM $=0.4 \mathrm{~nm}$ and reflectivity of $20 \%$; FBG2 has a central reflection wavelength equal to $1550.2 \mathrm{~nm}$, the same FWHM and a reflectivity of $70 \%$.

In Fig. 4a we can see that the spatial resolution $(\sim 1 \mathrm{~km})$ is too high and masks fibre spool L2. Although the spatial resolution used is equal to the length of spool L2, with the proposed OTDR method we can check if a fibre break occurs in spool L2 and differentiate it from a fibre cut near the end of spool L1. To perform this, the OTDR is tuned to each one of the central reflection wavelengths of the FBGs successively; the presence of the reflective events caused by the FBGs in Fig. 4b and Fig. 4c indicates that there are no failures in spools L0, L1 and L2.

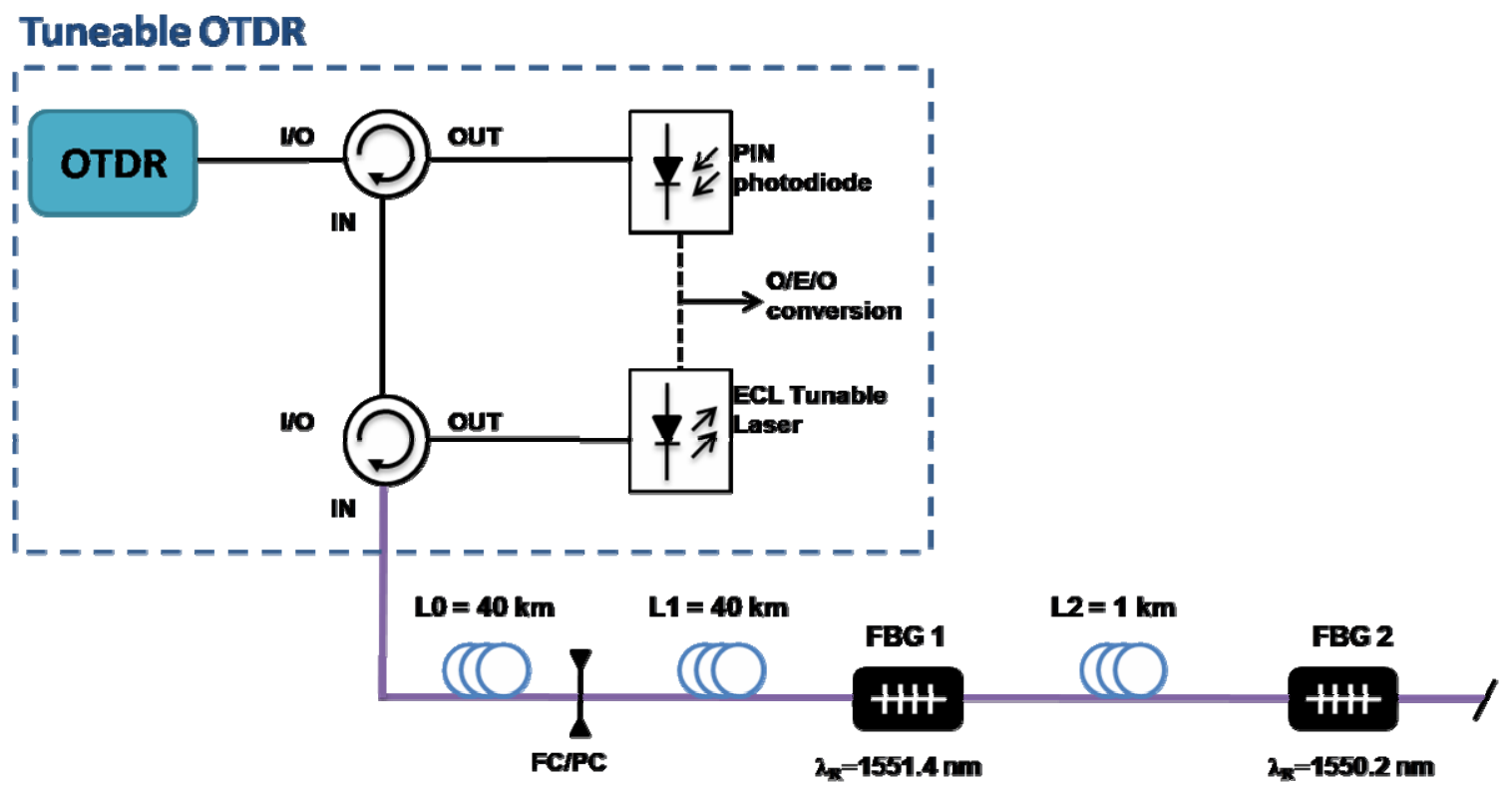

Fig. 3. Experimental setup: the OTDR is based on a tuneable ECL laser. A pulse width of $10 \mu$ s and a pulse repetition rate of $1 \mathrm{kHz}$ were used. 

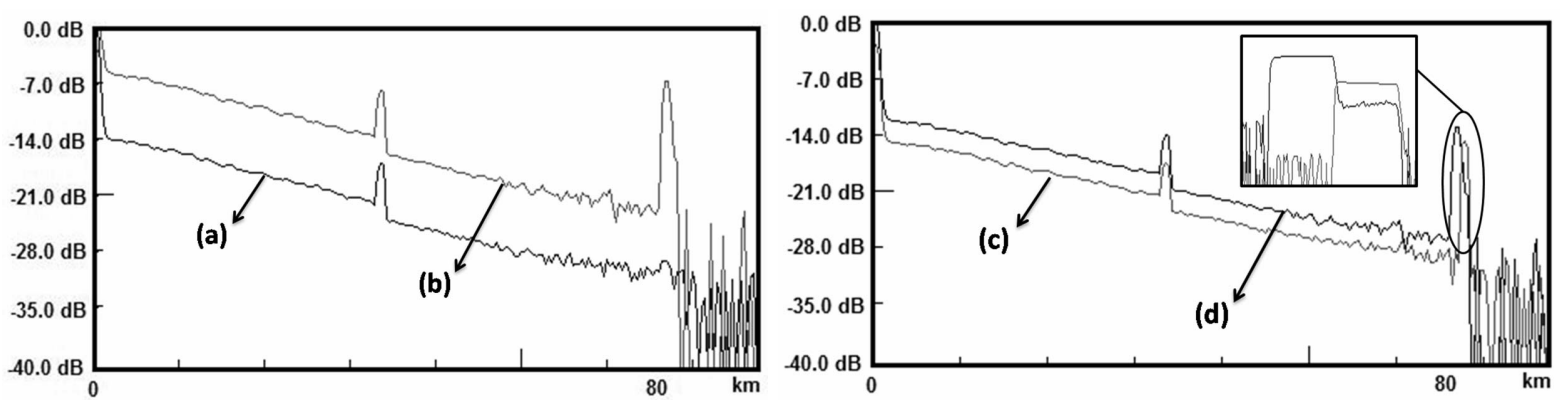

Fig. 4. (a) OTDR trace for $\lambda=1553.0 \mathrm{~nm}$ corresponding to conventional method, (b) OTDR trace for $\lambda=1551.4 \mathrm{~nm}$ corresponding to the central reflection wavelength of FBG1, (c) OTDR trace for $\lambda=1550.2 \mathrm{~nm}$ corresponding to the central reflection wavelength of FBG2, (d) same as (b). Inset is the zoomed area of the reflective events located at 80 and $81 \mathrm{~km}$ in both traces.

\section{CONCLUSIONS}

We propose a low-cost OTDR solution based on the addition of reflective elements in key intermediate and terminal locations applicable to any topology of PON network. By using a cheap tunable OTDR together with spectrally efficient set of multiple PS-FBGs, we can reduce initial investment costs while still maintaining reduced measurement time and/or receiver sensitivity requirements. We demonstrated that this solution based on successively monitoring fiber spans and simply checking the presence/absence of reflective events in both wavelength and time domains, allows to unambiguously determining the cause and location of fibre plant failures.

\section{ACKNOWLEDGEMENTS}

European FP7 EURO-FOS and SARDANA projects are acknowledged.

\section{REFERENCES}

[1] B. De Mulder et al.: "Nonintrusive fiber monitoring of TDM optical networks", J. Lightwave Technol., vol. 25, no. 1, pp. 305-317, Jan. 2007.

[2] H. Izumita et al.: Stochastic amplitude fluctuation in coherent OTDR and a new technique for its reduction by stimulating synchronous optical frequency hopping, J. Lightwave Technol., vol. 15, no. 2, pp. 267-278, Feb. 1997.

[3] S. Hann, J.-S. Yoo and C.-S. Park: Monitoring technique for a hybrid PS/WDM-PON by using a tunable OTDR and FBGs, Meas. Sci. Technol., vol. 17, pp. 1070-1074, Apr. 2006.

[4] K. Yuksel, V. Moeyaert, M. Wuilpart, and P. Mégret: Optical layer monitoring in passive optical networks (PONs): A review, in Proc. ICTON 2008, pp. 92-98, paper Tu.B1.1, 2008.

[5] R. Maciejko and L. Hadellis: Two signal processing enhancements for optical time domain reflectometry, J. Lightwave Technol., vol. 4, no. 5, pp. 538-546, May 1986.

[6] J. A. Lázaro, J. Prat, P. Chanclou, G. M. Tosi Beleffi, A. Teixeira, I. Tomkos, R. Soila, V. Koratzinos: Scalable extended reach PON, in. Proc. OFC/NFOEC 2008, San Diego, paper OTHL2, Feb. 2008. 